\title{
Магнетотранспортная спектроскопия интерфейсных, квантово-ямных и гибридных состояний в структурах с многочисленными слоями HgТе толщиной 16 нм
}

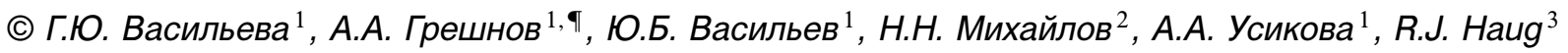 \\ ${ }^{1}$ Физико-технический институт им. А.Ф. Иоффе Российской академии наук, \\ 194021 Санкт-Петербург, Россия \\ ${ }^{2}$ Институт фризики полупроводников им. А.В. Ржанова \\ Сибирского отделения Российской академии наук, \\ 630090 Новосибирск, Россия \\ ${ }^{3}$ Institut fur Festkorperphysik, Leibniz Universitat Hannover, \\ D-30167 Hannover, Germany \\ ฯ E-mail: a_greshnov@hotmail.com
}

Поступила в Редакцию 6 декабря 2018 г.

В окончательной редакции 6 марта 2019 г.

Принята к публикации 15 марта 2019 г.

\begin{abstract}
Проведены измерения продольной и холловской компонент тензора сопротивления структур с многочисленными слоями $\mathrm{HgTe}$ толщиной 16 нм в магнитных полях до 12 Тл при температурах от 1.5 до $300 \mathrm{~K}$. Обнаружено, что наклон зависимости холловской компоненты от магнитного поля меняет знак при некоторой критической температуре, составившей $T_{c}=5$ и $10 \mathrm{~K}$ в двух исследованных образцах, что свидетельствует о наличии двух типов носителей и изменении соотношения между их вкладами в холловское сопротивление с температурой. Малость критической температуры и проявление „двухкомпонентного“ характера холловских кривых лишь при $T>T_{c}$ доказывают близость основного состояния системы к бесщелевому. При более высоких температурах $(20<T<200 \mathrm{~K})$ холловская концентрация с высокой точностью пропорциональна температуре. Описание законов дисперсии носителей в рамках 8-зонной $\mathbf{k p - м о д е л и ~ с ~ у ч е т о м ~ в ы з в а н н о г о ~ м е х а н и ч е с к и м и ~ н а п р я ж е н и я м и ~ р а с щ е п л е н и я ~ к р а я ~ з о н ы ~} \Gamma_{8}$, формирующей в HgTe состояния обоих типов, позволило количественно описать наблюдаемые особенности магнетотранспорта. Показано, что они связаны с параллельным заполнением электронных и дырочных состояний, образовавшихся в результате смешивания интерфейсных состояний, ответственных за фазу топологического изолятора, и квантово-размерных состояний зоны $\Gamma_{8}$.
\end{abstract}

Ключевые слова: магнетотранспортные характеристики, интерфейсные состояния, фазовая диаграмма, эффект Холла.

DOI: 10.21883/FTP.2019.07.47872.9040

\section{1. Введение}

Примером полупроводниковой структуры, в которой может быть реализована нулевая запрещенная зона, является квантовая яма $\mathrm{HgTe} / \mathrm{CdHgTe}$, где эффективная запрещенная зона определяется толщиной $\mathrm{HgTe}$ [1-4]. Кроме того, в таких структурах эффективную запрещенную зону можно сделать равной нулю за счет композиции сплава [5], температуры [6,7], деформации [8-10]. Во многих случаях закон дисперсии носителей при этом имеет дираковский вид $[2,11]$. В квантовых ямах $\mathrm{HgTe}$ наиболее активно изучались температурные зависимости сопротивления, и было показано, что температура может индуцировать фазовый переход из нормального состояния $\left(T>T_{c}\right)$ в топологически нетривиальное состояние вещества $\left(T<T_{c}\right)[12,13]$. Этот переход при некоторой критической температуре $T_{c}$ объяснялся сильной температурной зависимостью уровня $E 1$. Действительно, расчеты для квантовых ям HgТе показали, что температура индуцирует переход от полупроводника при комнатной температуре к топологическому изолятору (ТИ) при низкой температуре.
Составным квантовым ямам с нулевыми и инвертированными запрещенными зонами соответствует богатая фазовая диаграмма, содержащая полуметаллическое состояние, зонный изолятор и квантовые спиновые холловские изоляторы [14-16]. Созданные на основе этой системы сверхрешетки (СР) могут сильно отличаться по своим свойствам от квантовых ям, образующих слои СР. Было предсказано, что в таких слоистых структурах в результате взаимодействия между топологическими поверхностными модами могут возникать различные фазы, включая ТИ, полуметалл Дирака и полуметалл Вейля $[17,18]$. В зависимости от условий взаимодействия поверхностных мод ожидается проявление различных интересных физических эффектов. Также теоретически было показано, что фазовая диаграмма такой системы содержит топологическую фазу полуметалла Вейля простейшего типа, т.е. имеется только два узла Дирака противоположной хиральности, разделенных в пространстве импульсов. Экспериментальные исследования физических свойств дираковских фермионов в таких структурах нам не известны. В то 
же время исследования сверхрешеток $\mathrm{HgTe} / \mathrm{CdTe}$ активно проводились в (70-80)-х годах прошлого века главным образом в связи с их использованием в качестве инфракрасных фотоприемников. Уже тогда в расчетах зонной структуры этих сверхрешеток были получены свидетельства существования интерфейсных состояний [19]. Влияние этих состояний на свойства одиночных и двойных квантовых ям $\mathrm{HgTe} / \mathrm{CdHgTe}$ было изучено в транспортных и оптических экспериментах, однако вплоть до настоящего времени об экспериментальных исследованиях многослойных структур не сообщалось.

В настоящей работе экспериментально и теоретически изучены магнетотранспортные характеристики многослойных структур $\mathrm{HgTe} / \mathrm{CdHgTe}$, изготовленных на основе теллурида ртути с инвертированной зонной структурой (толщина слоев HgТе превышает критическую толщину 6.3 нм). Исследовано поведение системы в диапазоне температур от 1.5 до $200 \mathrm{~K}$, обнаруженные особенности магнетотранспорта объяснены при помощи расчетов энергетических спектров в рамках 8-зонной kp-модели с учетом механических напряжений, возникающих из-за существенного рассогласования постоянных решетки HgTe и CdTe.

\section{2. Эксперимент}

Образцы выращивались методом молекулярно-пучковой эпитаксии [20]. В качестве подложки использовались пластины GaAs толщиной 400 мкм, на которые наносились слои ZnTe (30 нм) и CdTe (6 мкм). Были выращены

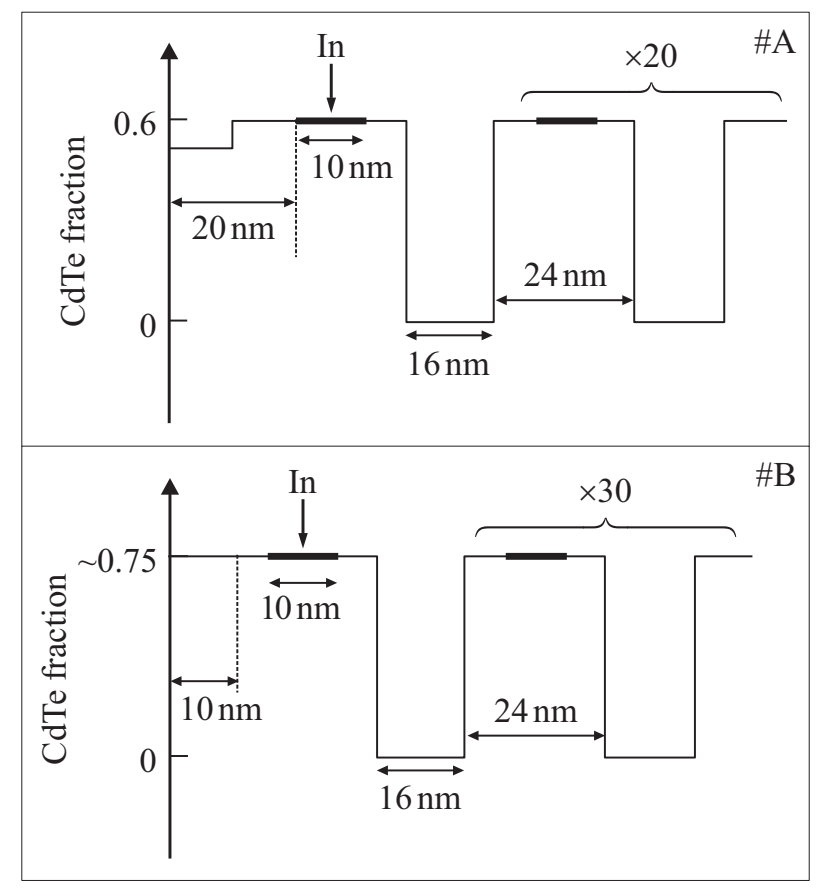

Рис. 1. Зонная диаграмма исследованных структур $\mathrm{HgTe} / \mathrm{CdHgTe} \mathrm{A} \mathrm{и} \mathrm{B.}$

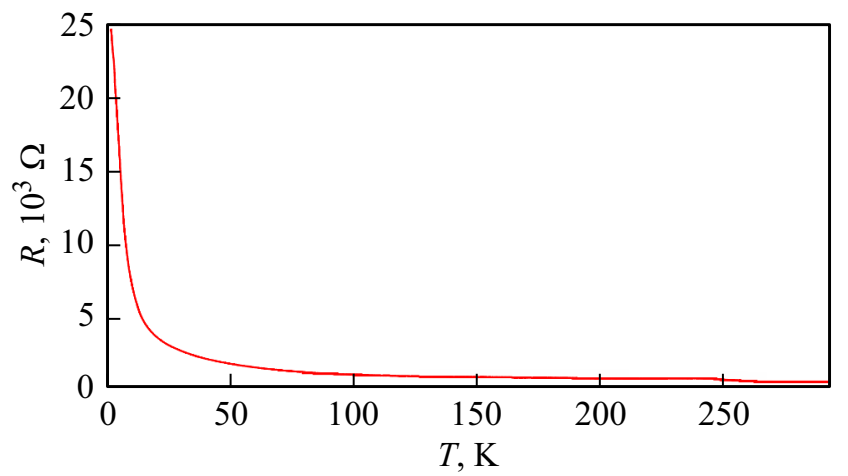

Рис. 2. Температурная зависимость сопротивления структуры А.

две однотипные многослойные структуры. Структура А содержала 20 квантовых ям НgТе толщиной 16 нм, структура В - 30 квантовых ям толщиной 16 нм, разделенных барьерами CdHgTe (рис. 1). Из этих структур были изготовлены мостики Холла литографическим методом с размерами $L \times W=1000 \times 200$ мкм. Измерения проводились четырехзондовым методом. На образец подавался переменный ток. В качестве источника тока использовался Keithly 6221. Сигнал снимался через синхронный усилитель. Используемая частота переменного тока была равна 17.7 Гц. Измерения продольной и холловской компонент тензора сопротивления проводились в магнитных полях вплоть до 12 Тл в диапазоне температур от 1.5 до $300 \mathrm{~K}$. При низких температурах наблюдались осцилляции продольного сопротивления и проявление квантового эффекта Холла, что свидетельствует о достаточно высоком качестве образцов.

На рис. 2 показано удельное сопротивление $R$ структуры А как функция температуры. Аналогичная зависимость получена и для структуры В (не показана). Видно, что сопротивление увеличивается с понижением температуры, и в температурном интервале от 40 до $1.5 \mathrm{~K}$ степень изменения сопротивления с температурой резко возрастает, так что рост сопротивления происходит более чем на порядок. Для выявления причин такого поведения были проведены измерения эффекта Холла (на рис. 3 показаны данные для структуры В) и определены температурные зависимости концентрации носителей $n$ (рис. 4), которые оказались близкими к линейным в интервале температур $T=20-200$ К. При этом с понижением температуры холловские кривые сильнее отклоняются от линейных, показывая в температурном интервале $12-80 \mathrm{~K}$ немонотонное поведение, характерное для систем с двумя типами носителей (полуметаллов). При еще более низких температурах происходит изменение знака холловского напряжения $\left(T_{c}=10 \mathrm{~K}\right.$ для образца А и $5 \mathrm{~K}$ для образца В), что указывает на изменение типа проводимости и переход через состояние, близкое к бесщелевому. 

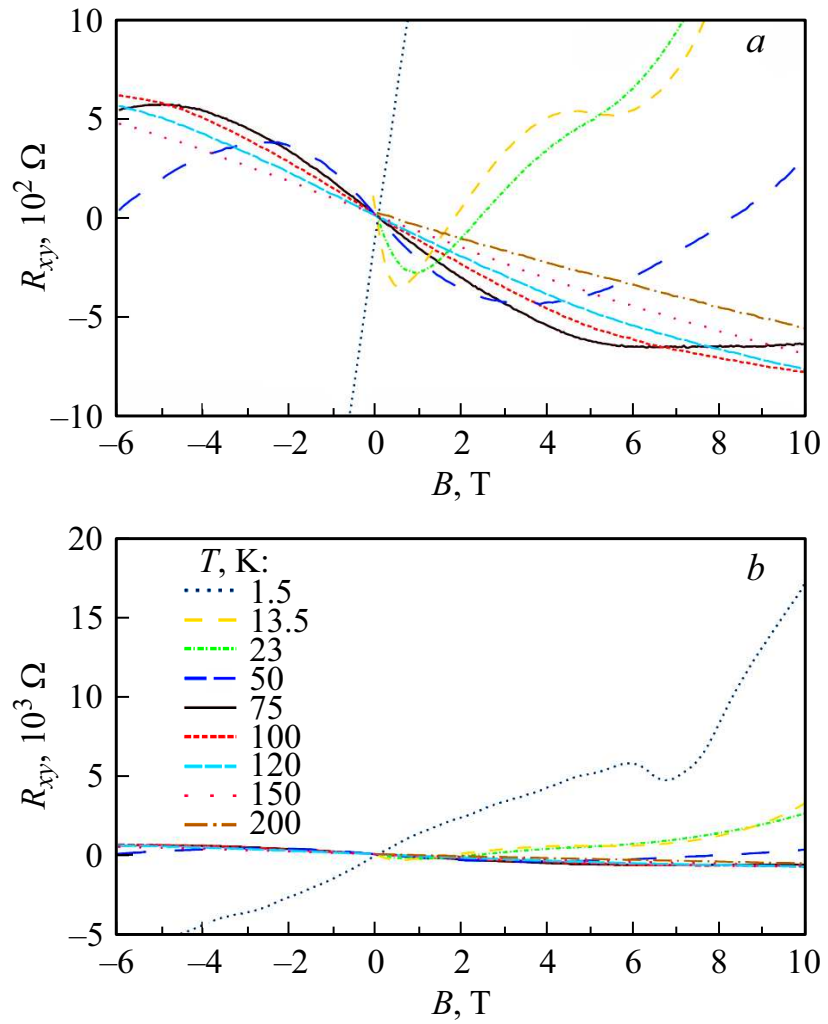

Рис. 3. Холловское сопротивление структуры В в температурном интервале $1.5-200 \mathrm{~K}$ в масштабе до $1(a), 20$ кОм $(b)$.

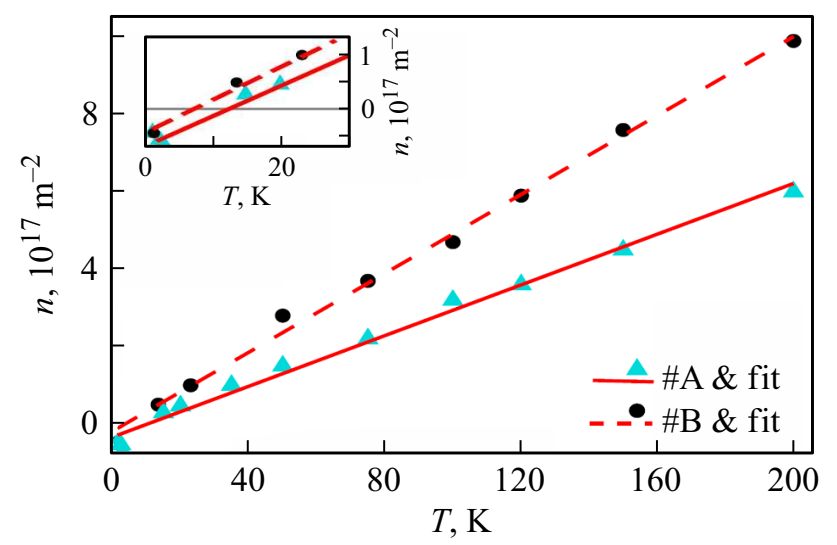

Рис. 4. Температурные зависимости суммарной холловской концентрации в структурах А и В в интервалах 0-200 и 0-30 K (на вставке).

\section{3. Теория и обсуждение}

Для объяснения экспериментально наблюдаемых особенностей магнетотранспорта мы провели расчеты энергетического спектра носителей в исследуемых структурах в рамках 8-зонного кр-метода [14]. При этом возможность туннелирования через барьер $\mathrm{CdHgTe} \mathrm{ши-}$ риной 24 нм с образованием сверхрешеточной минизоны не учитывалась как чрезвычайно малая, и за- дача сводилась к рассмотрению энергетического спектра напряженной квантовой ямы $\mathrm{HgTe}$ шириной 16 нм. Учет механических напряжений, возникающих из-за существенного рассогласования $(0.3 \%)$ постоянных решетки материалов $\mathrm{HgTe}$ и $\mathrm{CdHgTe}$, является ключевым фактором $[21,22]$ в образовании полуметаллического состояния, проявляющегося в виде немонотонного, „двухкомпонентного“ поведения зависимости холловской компоненты тензора сопротивления от магнитного поля [9], хорошо заметного на рис. 3 в интервале температур от 10 до $50 \mathrm{~K}$. В расчете использованы зонные параметры Кейна $(P)$ и Латтинжера $\left(\gamma_{1,2,3}\right)$, близкие к использованным в работе [21], и простейшая симметрия структуры (001), не препятствующая проявлению полуметаллических свойств [22]. При такой симметрии единственной значимой „деформационной“ поправкой в 8-зонный $\mathbf{k p - г а м и л ь т о н и а н ~ я в л я е т - ~}$ ся расщепление края энергетической зоны $\Gamma_{8}$, причем знак деформационного потенциала и вид деформации слоев HgТе обеспечивают смещение вниз состояний тяжелых дырок, а электронные состояния (играющие при обычной, не инвертированной, зонной структуре роль легких дырок) смещаются вверх на некоторую величину $\Delta V$. В расчетах мы использовали значение $\Delta V=7$ мэВ, что соответствует приблизительно половине от полного эффекта механических напряжений между слоями $\mathrm{HgTe}$ и $\mathrm{CdTe}$ для структуры ориентации (001) (в [22] указывается, что расчеты зонной структуры проводились при учете лишь $48.5 \%$ оцененных механических напряжений) и согласуется с составом барьерных слоев и возможностью релаксации напряжений. Результаты расчетов зонной структуры в виде зависимости энергии от квадрата волнового вектора, $E\left(q^{2}\right)$, представлены на рис. 5 для ориентаций волнового вектора в плоскости под углами $0,15,30$ и $45^{\circ}$ к кристаллографическим осям.

Прежде всего обсудим природу формирования электронных и дырочных состояний, дисперсия которых изображена на рис. $5, a$, в исследуемой системе, содержащей многочисленные слои полупроводника $\mathrm{HgTe}$ с инвертированной зонной структурой шириной 16 нм и интерфейсы с барьерными слоями $\mathrm{CdHgTe}$, описываемыми обычной схемой зон. „Визитной карточкой“ такого интерфейса [23] являются локализованные состояния, располагающиеся в глубине запрещенной зоны полупроводника с инвертированной зонной структурой $(\mathrm{HgTe})$, их положение для одиночной гетерограницы это $\sim 90$ мэВ ниже края зоны $\Gamma_{8}[14]$, который мы приняли за точку отсчета энергии. Конечное перекрытие экспоненциальных хвостов волновой функции левого и правого интерфейсов квантовой ямы приводит к формированию симметричной и антисимметричной комбинаций таких состояний и расщеплению уровня энергии на два, причем антисимметричное состояние обладает положительной дисперсией и смещается по энергии вверх при сужении квантовой ямы и увеличении модуля волнового вектора $q$. Однако при конечной величине $q$ 

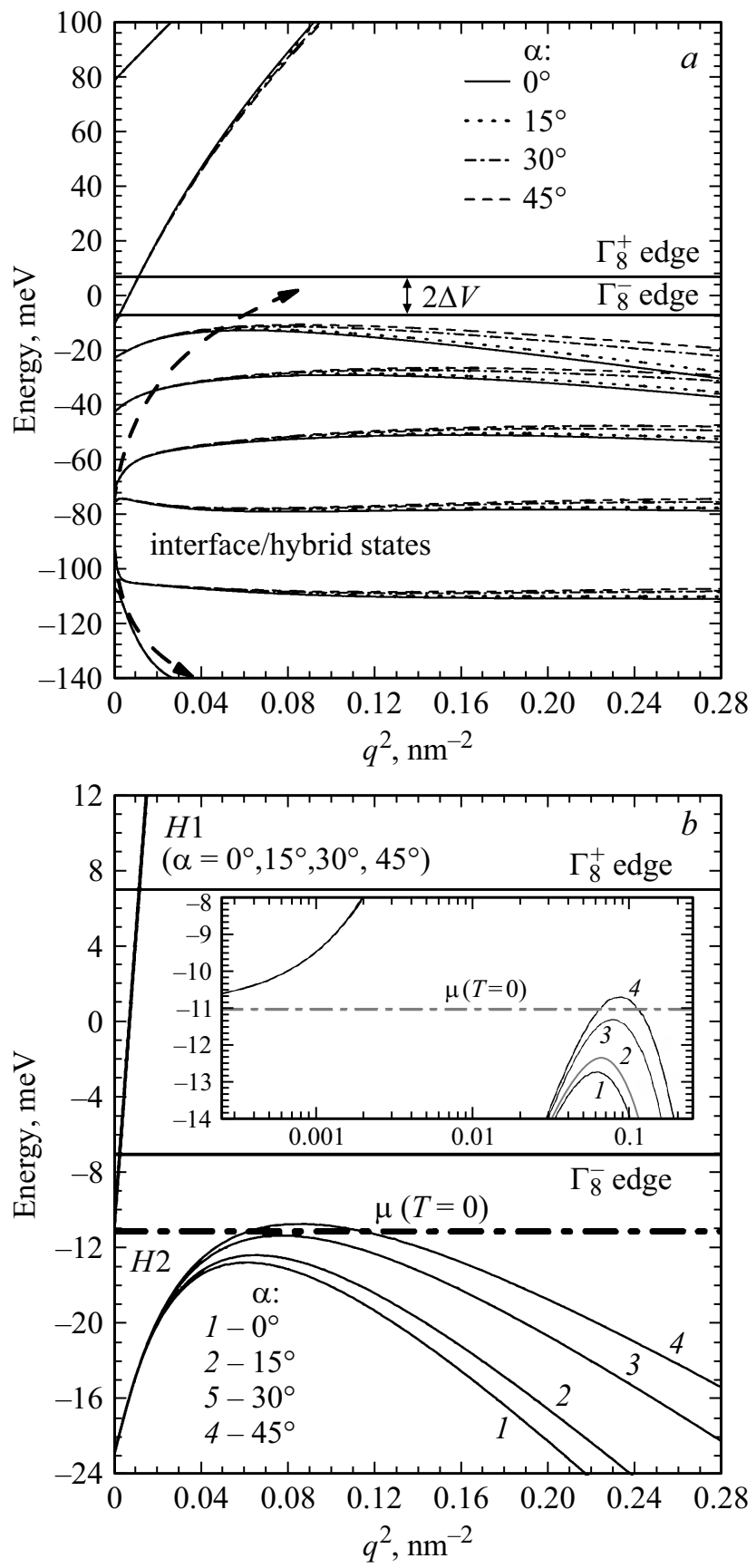

Рис. 5. Зависимости энергии носителей от квадрата двумерного волнового вектора для различных его ориентаций $(\alpha-$ угол относительно главного кристаллографического направления $\langle 10\rangle)$ для структуры симметрии (001) со слоями $\mathrm{HgTe}$ толщиной 16 нм при наличии деформационного расщепления зоны $\Gamma_{8}$ в размере $2 \Delta V=14$ мэВ: $a-$ общая картина дисперсии, $b-$ дисперсионные ветви вблизи уровня Ферми (на вставке крупно показано перекрытие электронной и дырочной подзон).

интерфейсное состояние приобретает компоненту волновой функции тяжелых дырок и возникает зацепление (coupling) с квантово-размерными состояниями тяжелых дырок, обладающих изначально отрицательной дисперсией. В результате имеет место гибридизация таких состояний, а итоговая дисперсия становится немонотонной для всех ветвей, отвечающих состояниям тяжелых дырок при $q=0$, помимо самого верхнего $(n=1)$ и лежащих по энергии ниже интерфейсного состояния. При этом дисперсионная ветвь, начинающаяся из основного уровня размерного квантования тяжелых дырок, имеет исключительно положительную дисперсию, уже при малых $q$ проходит край зоны $\Gamma_{8}$ и далее переходит в первое квантово-размерное состояние электронов (волновая функция которых при обычной зонной структуре соответствует легким дыркам).

Наличие ветвей с немонотонной дисперсией приводит к возможности перекрытия по энергии, а значит, и одновременного заполнения состояний с противоположной дисперсией (электронов и дырок). Экспериментально такая ситуация реализуется благодаря механическим напряжениям, возникающим в системе $\mathrm{HgTe} / \mathrm{CdHgTe}$ из-за существенного рассогласования постоянных решетки $[21,22]$. В силу деформационных напряжений край зоны $\Gamma_{8}$ расщепляется на величину $2 \Delta V \approx 15$ мэВ, причем для состояний тяжелых дырок смещение происходит вниз по оси энергий, а для состояний легких дырок (значит, и интерфейсных) - вверх. В результате „выталкивание“ ветвей дисперсии тяжелых дырок вверх интерфейсными состояниями возможно вплоть до положительной энергии $+\Delta V$, в то время как дисперсия „электронной“ ветви начинается с энергии $-\Delta V-E_{0}$, где $E_{0}$ - энергия основного уровня размерного квантования тяжелых дырок. Перекрытие электронных и дырочных состояний в некотором энергетическом интервале внутри указанного приводит к полуметаллическому характеру электронного транспорта [9].

В образцах, исследованных в данной работе, полуметаллические свойства не проявляются при температурах ниже $5 \mathrm{~K}$, поэтому перекрытие зон в основном состоянии отсутствует или является ничтожным. Мы полагаем, что зонная структура и положение уровня Ферми $\mu$ близки к представленным в деталях на рис. $5, b$, так что при наиболее низких температурах имеется небольшая концентрация дырок с преимущественным направлением волнового вектора вдоль $\langle 11\rangle$, а электроны отсутствуют. Экспериментально это проявляется в виде стандартного (монотонного) хода холловской зависимости образца В при $T=1.5 \mathrm{~K}$ (рис. 3,a), соответствующего суммарной двумерной концентрации дырок $p_{t}=p_{0} \approx 5 \cdot 10^{11} \mathrm{~cm}^{-2}$. С учетом общего количества слоев $\mathrm{HgTe}$ в структуре расчетная концентрация на один слой составляет $p_{1} \approx 2 \cdot 10^{10} \mathrm{~cm}^{-2}$, однако квантовая особенность в поведении холловского сопротивления $R_{x y}(B)$ в магнитных полях $B \approx 6-7$ Тл определенно связана с достижением частью носителей фактора заполнения $v=1$, соответствующего в таком магнитном поле концентрации $\sim 10^{11} \mathrm{~cm}^{-1}$. Мы полагаем, что действительно такова концентрация дырок в 4-5 слоях НgТе, формирующих ступеньку квантового эффекта Холла, в то время как в остальных слоях концентрация близка к нулю и их вклад в транспорт 
является ничтожным. Причиной этого может служить релаксация механических напряжений от слоя к слою, встроенный электростатический потенциал и другие факторы, учитывая, насколько чувствительной к изменению параметров является картина зонной структуры вблизи уровня Ферми.

С другой стороны, при высоких температурах, составляющих на энергетической шкале единицы и десятки мэВ, уровень Ферми перемещается из области дырочных состояний в область электронной дисперсии, так как в противном случае температурное заполнение дырочных состояний вблизи максимума „гибридной“ дисперсии привело бы при $T \approx 100 \mathrm{~K}$ к „гигантской“ концентрации дырок, $p_{t}=30 p_{1} \approx 3 \cdot 10^{13} \mathrm{~cm}^{-2}$, на 2 порядка превышающей первоначальную $p_{0}$. Поэтому при таких температурах уровень Ферми лежит глубоко в зоне электронов, так что

$$
\mu(T) \approx \mu(t=0)+\alpha T
$$

с $\alpha>1$ (по нашим оценкам $\alpha \approx 2$ ), при этом концентрации электронов и дырок приблизительно равны и значительно (на порядок) превышают $p_{0}$. Это означает, что система находится в полуметаллическом режиме, где эффект Холла может быть знакопеременным, при этом в пределах сильного и слабого магнитного полей холловское сопротивление пропорционально магнитному полю, но с разными эффективными концентрациями, равными соответственно

$$
\begin{gathered}
n_{\text {high }}=n-p, \\
n_{\text {low }}=\frac{\left(\mu_{n} n+\mu_{p} p\right)^{2}}{\mu_{n}^{2} n-\mu_{p}^{2} p} .
\end{gathered}
$$

Поскольку массы электронов и дырок в рассматриваемой системе, а вместе с ними и подвижности $\mu_{n}, \mu_{p}$ различаются более чем на порядок, из формулы (3) следует, что концентрация $n_{\text {low }}$ может существенно отличаться от $n$ лишь в случае, если $p$ превосходит $n$ хотя бы на порядок величины. Поэтому при температурах $T>20 \mathrm{~K}$ можно полагать $n_{\text {low }}=n$, в результате $n_{\text {high }}$ и $n_{\text {low }}$ имеют разные знаки, а эффект Холла является знакопеременным, что экспериментально наблюдалось в интервале температур от 10 до $50 \mathrm{~K}$ (рис. 3). При больших температурах от знакопеременности остается немонотонность холловской зависимости $(T=75 \mathrm{~K})$ или даже ее небольшая нелинейность $(T=100-200 \mathrm{~K})$, что объясняется перемещением точки кроссовера (перемены режима) в область все больших магнитных полей изза линейно возрастающего с температурой отношения $n /(p-n)$, где $p-n=p_{0}$. Таким образом, извлеченная из эффекта Холла концентрация на рис. 4 - это концентрация носителей, заполняющих первую электронную ветвь. Как видно из рис. 5, $b$, эта ветвь дисперсии, стартующая вверх с первого уровня размерного квантования тяжелых дырок, строго следует квадратичному закону

$$
E(q)=\frac{\hbar^{2} q^{2}}{2 m_{n}}
$$

с $m_{n} \approx 0.025 m_{0}$ на протяжении нескольких десятков мэВ, формируя не зависящую от энергии плотность состояний. В результате заполнения постоянной плотности электронных состояний с температурой формируется концентрация

$$
n(T)=\frac{m_{n} \alpha T}{\pi \hbar^{2}}
$$

которая и проявляется в эффекте Холла (рис. 4) и температурной зависимости продольной компоненты тензора сопротивления (рис. 2).

В заключение обратим также внимание на квантовую особенность, формирующуюся на холловской кривой, соответствующей температуре $T=13.5 \mathrm{~K}$, в районе $B \approx 5$ Тл (рис. 3,a). Поскольку ее появление в рассматриваемой системе связано с достижением одной из групп носителей фактора заполнения $v=1$ (фундаментального плато квантового эффекта Холла), отношение кванта сопротивления $e^{2} / h(\sim 26$ кОм $)$ к наблюдаемой величине $R_{x y} \approx 0.5$ кОм позволяет оценить число слоев $\mathrm{HgTe,} \mathrm{вовлеченных} \mathrm{в} \mathrm{формирование} \mathrm{особенности,}$ которое составляет $\sim 50$ и превышает число слоев в структуре (30). Однако в рассматриваемом режиме сопротивление определяется двумя группами носителей, в результате диссипативная составляющая тензора проводимости сравнима по величине с холловской, что в $\sim 2$ раза уменьшает положение формирующегося плато квантового эффекта Холла, но не препятствует наблюдению особенности.

\section{Благодарности}

Г.Ю. Васильева благодарит РНФ, грант № 17-72-10134.

\section{Конфликт интересов}

Авторы заявляют, что у них нет конфликта интересов.

\section{Список литературы}

[1] M. Schultz, U. Merkt, A. Sonntag, U. Rossler, R. Winkler, T. Colin, P. Helgesen, T. Skauli, S. Løvold. Phys. Rev. B, 57, 14772 (1998).

[2] B. Buttner, C.X. Liu, G. Tkachov, E.G. Novik, C. Brüne, H. Buhmann, E.M. Hankiewicz, P. Recher, B. Trauzettel, S.C. Zhang, L.W. Molenkamp. Nature Phys., 7, 418 (2011).

[3] М.С. Жолудев, Ф. Теп, С.В. Морозов, М. Орлита, К. Консейон, С. Руфенах, В. Кнап, В.И. Гавриленко, С.А. Дворецкий, Н.Н. Михайлов. Письма ЖЭТФ, 100, 895 (2014).

[4] J. Ludwig, Yu.B. Vasilyev, N.N. Mikhailov, J.M. Poumirol, Z. Jiang, O. Vafek, D. Smirnov. Phys. Rev. B, 89, 241406(R) (2014).

[5] E.G. Novik, A. Pfeuffer-Jeschke, T. Jungwirth, V. Latussek, C.R. Becker, G. Landwehr, H. Buhmann, L.W. Molenkamp. Phys. Rev. B, 72, 035321 (2005). 
[6] S. Wiedmann, A. Jost, C. Thienel, C. Brüne, P. Leubner, H. Buhmann, L.W. Molenkamp, J.C. Maan, U. Zeitler. Phys. Rev. B, 91, 205311 (2015).

[7] A.M. Kadykov, S.S. Krishtopenko, B. Jouault, W. Desrat, W. Knap, S. Ruffenach, C. Consejo, J. Torres, S.V. Morozov, N.N. Mikhailov, S.A. Dvoretskii, F. Teppe. Phys. Rev. Lett., 120, 086401 (2018).

[8] P. Leubner, L. Lunczer, C. Brüne, H. Buhmann, L.W. Molenkamp. Phys. Rev. Lett., 117 (8), 086403 (2016).

[9] З.Д. Квон, Е.Б. Ольшанецкий, Д.А. Козлов. Письма ЖЭТФ, 87, 588 (2008).

[10] Д.А. Козлов, З.Д. Квон, Н.Н. Михайлов и др. Письма ЖЭТФ, 93, 186 (2011).

[11] B.A. Bernevig, T.L. Hughes, S.-C. Zhang. Science, 314, 1757 (2006).

[12] G.M. Gusev, Z.D. Kvon, E.B. Olshanetsky, A.D. Levin, Y. Krupko, J.C. Portal, N.N. Mikhailov, S.A. Dvoretsky. Phys. Rev. B, 89, 125305 (2014).

[13] M. Marcinkiewicz, S. Ruffenach, S.S. Krishtopenko, A.M. Kadykov, C. Consejo, D.B. But, W. Desrat, W. Knap, J. Torres, A.V. Ikonnikov, K.E. Spirin, S.V. Morozov, V.I. Gavrilenko, N.N. Mikhailov, S.A. Dvoretskii, F. Teppe. Phys. Rev. B, 96, 035405 (2017).

[14] А.А. Грешнов, Ю.Б. Васильев, Н.Н. Михайлов, Г.Ю. Васильева, Д. Смирнов. Письма ЖЭТФ, 97 (2), 108 (2013).

[15] J. Ruan, S.-K. Jian, H. Yao, H. Zhang, S.-C. Zhang, D. Xing. Nature Commun., 7, 11136 (2016).

[16] T. Rauch, S. Achilles, J. Henk, I. Mertig. Phys. Rev. B, 96, 035124 (2017)

[17] A.A. Burkov, L. Balents. Phys. Rev. Lett., 107, 127205 (2011).

[18] G.B. Halász, L. Balents. Phys. Rev. B, 85, 035103 (2012).

[19] M. Voos. Surf. Sci. Reports, 7, 189 (1987).

[20] Yu.G. Sidorov, S.A. Dvoretsky, M.V. Yakushev, N.N. Mikhailov, V.S. Varavin, V.I. Liberman. Thin Sol. Films, 306, 253 (1997).

[21] Z.D. Kvon, E.B. Olshanetsky, E.G. Novik, D.A. Kozlov, N.N. Mikhailov, I.O. Parm, S.A. Dvoretsky. Phys. Rev. B, 83, 193304 (2011).

[22] E.B. Olshanetsky, Z.D. Kvon, N.N. Mikhailov, E.G. Novik, I.O. Parm, S.A. Dvoretsky. Sol. St. Commun., 152, 265 (2012).

[23] М.И. Дьяконов, А.В. Хаецкий. Письма ЖЭТФ, 33, 115 (1981).

\section{Magnetotransport spectroscopy of interface, quantum well, and hybrid states in structures with multiple $\mathrm{HgTe} 16 \mathrm{~nm}$ thick layers}

\author{
G.Yu. Vasilyeva ${ }^{1}$, A.A. Greshnov ${ }^{1}$, Yu.B. Vasilyev ${ }^{1}$, \\ N.N. Mikhailov' ${ }^{2}$, A.A. Usikova ${ }^{1}$, R.J. Haug ${ }^{3}$ \\ ${ }^{1}$ loffe Institute, \\ 194021 St. Petersburg, Russia \\ ${ }^{2}$ Rzhanov Institute of Semiconductor Physics, \\ Siberian Branch of Russian Academy of Sciences, \\ 630090 Novosibirsk, Russia \\ ${ }^{3}$ Institut fur Festkorperphysik, \\ Leibniz Universitat Hannover, \\ D-30167 Hannover, Germany
}

Abstract Measurements of the longitudinal and Hall resistance tensor components were made in structures with multiple $\mathrm{HgTe}$ layers of $16 \mathrm{~nm}$ width in magnetic fields up to $12 \mathrm{~T}$ at the temperatures from 1.5 to $300 \mathrm{~K}$. It was found that the slope of the magnetic field dependence of the Hall resistance changes its sign at some critical temperature which is $T_{c}=5$ and $10 \mathrm{~K}$ in the two samples studied indicating the presence of two types of carriers and the change in the ratio between their contributions to the Hall resistance with temperature. The small critical temperature and the manifestation of the "two-component" character of the Hall curves only at $T>T_{c}$ prove that the ground state of the system at $T=T_{c}$ is a gapless one. At higher temperatures $(20<T<200 \mathrm{~K})$, the Hall concentration with good accuracy is proportional to the temperature. The description of the carrier dispersion laws by the 8-band kp-model taking into account the splitting of the $\Gamma_{8}$ band edge caused by mechanical stresses, which forms both types of state in $\mathrm{HgTe}$, allowed us to quantitatively describe the observed magnetotransport features. It was shown that they are associated with simultaneous filling of electron and hole states formed as a result of mixing the interface states responsible for the phase of the topological insulator and quantum-dimensional states in the $\Gamma_{8}$ band. 